\title{
Caracterización de la movilidad metropolitana de Málaga mediante la telefonía móvil. Estudio comparativo frente a encuestas
}

\author{
N. Cáceres ${ }^{1}$, L.M. Romero² ${ }^{2}$ F.G. Benítez ${ }^{2}$, A. Reyes ${ }^{2}$, C. Ruiz de Alarcón², \\ F.J. Morales ${ }^{2}$ \\ ${ }^{1}$ Ingeniería de los Transportes, AICIA, Sevilla, España \\ ${ }^{2}$ Ingeniería e Infraestructura de los Transportes. Universidad de Sevilla, España
}

\section{RESUMEN}

El análisis de la movilidad en una determinada región es un aspecto esencial en las tareas de planificación del transporte, siendo vital manejar información actualizada a fin de que sus resultados sean más dinámicos y realistas. Recientemente se ha producido un gran desarrollo en la sensorización del tráfico, ligado tanto a la propia infraestructura como al vehículo, surgiendo cada vez más fuentes de datos provenientes de otras actividades que pueden enriquecer la información de movilidad existente. En este sentido la telefonía móvil se perfila como alternativa con alto potencial para la recopilación de datos de movilidad. El propio funcionamiento de estos sistemas genera ciertos registros espacio-temporales que, debidamente tratados y encriptados sin revelar información confidencial de sus abonados, permitiría inferir datos relativos a la dinámica de actividad humana de forma transparente. Sin embargo, esta fuente de información procede de una tecnología diseñada con otra finalidad, por lo que debe ser cuidadosamente analizada como paso previo a su uso en la caracterización completa de la movilidad de una zona.

Esta comunicación evalúa el potencial de la información derivada de la telefonía móvil como complemento de las metodologías tradicionales en futuros estudios de transporte. El estudio piloto, desarrollado en el marco del proyecto DIURMOVIL (AOPJA, 2015), ha llevado a cabo un análisis detallado de la movilidad en el área urbana y metropolitana de Málaga mediante datos provenientes de la tecnología móvil, contrastando los resultados con fuentes tradicionales de información basadas en encuestas sobre el mismo área de estudio. Todo ello ha permitido identificar las potencialidades y limitaciones de esta tecnología, atendiendo a la situación actual del sector, valorando aspectos clave en la realización de estudios de transporte.

\section{INTRODUCCIÓN}

\subsection{Objetivos}

El análisis cualitativo y cuantitativo de la movilidad en una determinada región es un aspecto esencial en las tareas de planificación del transporte en dicho área de estudio. Con el estado actual de la técnica, la estimación y análisis de la movilidad requiere de grandes campañas 
de recopilación de datos que consumen importantes recursos económicos, humanos y temporales, por lo que su ejecución no suele abordarse con la frecuencia deseada. Durante la última década se ha producido un gran desarrollo en la sensorización del tráfico surgiendo cada vez más fuentes de datos provenientes de otras actividades que enriquecen la información existente en cantidad y calidad. Por separado, estas fuentes carecen de las características y potencial que un sistema que lo integrara en su conjunto podría ofrecer a los estudios de transporte. En este sentido, la telefonía móvil se ha posicionado en los últimos años como alternativa de alto potencial para la recopilación de datos de movilidad fiables y actualizados. Su extendido uso en las sociedades modernas permite considerar a sus abonados como una muestra extensa de datos relevantes para la caracterización de la movilidad; la tasa de penetración en España supera ya el 108\% (CNMC, 2015). El avance de esta tecnología ha propiciado la aparición de nuevas funcionalidades que permiten generar "huellas" espacio-temporales de desplazamientos de usuarios; todo ello debidamente agregado y encriptado, sin revelar información confidencial. Sin embargo, estas fuentes de información proceden de sistemas diseñados con otro fin, por lo que presentan también una serie de limitaciones que impiden su uso por sí solas en la caracterización de la movilidad de una zona.

Esta comunicación evalúa y pondera los resultados derivados de una comparativa exhaustiva de ambas fuentes: telefonía móvil y encuestas, llevada a cabo en el ámbito del proyecto DIURMOVIL (AOPJA, 2015). En los siguientes párrafos se detallan las principales conclusiones derivadas del proyecto, especialmente en relación con el potencial de la telefonía como alternativa a metodologías tradicionales en futuros estudios de movilidad.

\subsection{Fuentes de información}

Para el análisis comparativo se emplean matrices O-D derivadas tanto de la telefonía móvil como de encuestas, sobre la aglomeración urbana de Málaga, la cual se posiciona en quinto lugar por peso demográfico de un total de 85 grandes áreas urbanas españolas con más de 50.000 habitantes (Grandes Áreas Urbanas Españolas, 2012). Además de la ciudad de Málaga, el área de estudio lo conforman los municipios de Alhaurín de la Torre, Alhaurín el Grande, Almogía, Álora, Benalmádena, Cártama, Casabermeja, Colmenar, Mijas, Pizarra, Rincón de la Victoria y Totalán; junto con Torremolinos y Fuengirola. Este área se dividió en 178 zonas de transporte, que a su vez fueron agregadas en áreas más generales basadas en zonas de transporte adyacentes para formar un total de 46 macrozonas.

Adicionalmente se ha contado con las tradicionales encuestas domiciliarias de movilidad; en particular con la llevada a cabo en 2010 por el Consorcio de Transporte Metropolitano del Área de Málaga (CTMAM) con el objetivo de tener una visión detallada de los patrones de viaje realizados por los residentes del área de estudio. Los resultados han sido estadísticamente expandidos y validados, generando las matrices O-D con los viajes realizados en un día laborable medio de invierno en el área del estudio. Por otro lado, se ha 
dispuesto de matrices derivadas de datos de la telefonía móvil. Por lo general, estos datos provienen de los operadores de redes móviles que recogen, almacenan y procesan grandes cantidades de eventos generados por los usuarios del servicio. En este estudio, el operador fue Telefónica, uno de los principales proveedores que operan en España con una cuota de mercado del 40\% (CNMC, 2015). Las matrices O-D fueron creadas por una herramienta de análisis de datos llamada Smart Steps desarrollada por Telefónica Dynamic Insights (Smart Steps, 2015). Smart Steps procesa millones de eventos móviles generados por los usuarios en la red de Telefónica, con el fin de disponer de "huellas" agregadas y anónimas de la movilidad de usuarios. Estos eventos consisten en interacciones activas con la red, relacionados con llamadas telefónicas y mensajes de texto, así como interacciones pasivas asociadas con la pérdida/restablecimiento de cobertura, cambios de grupos de antenas, y registros periódicos que el sistema realiza cada 4 horas. A través del análisis de eventos o "huellas", SmartSteps identifica patrones de movilidad. Conviene destacar que los movimientos siempre se consideran como un todo, para garantizar la privacidad. Este tratamiento agregado sólo permite conocer las tendencias de las multitudes pero nunca de los individuos. A ello le sigue un extrapolado estadístico para realizar una estimación sobre el total del universo. Siguiendo este enfoque, Smart Steps ha analizado los eventos móviles generados sobre el área de estudio durante dos semanas en febrero de 2015, con objeto de estimar las pautas de movilidad de la población sobre la base de la misma zonificación empleada en la encuesta. Así, se generaron las matrices que representan los viajes realizados en un día laborable medio de invierno sobre el área.

\section{TELEFONÍA MÓVIL COMO FUENTE DE INFORMACIÓN DE MOVILIDAD}

Con objeto de determinar las potencialidades y limitaciones, se ha realizado un análisis del uso de la telefonía móvil como fuente de información de movilidad, estructurando las ventajas competitivas de esta tecnología respecto a la situación actual de las metodologías tradicionales. Cada uno de los aspectos incluidos en dicho análisis es abordado en las siguientes secciones.

\subsection{Consideraciones previas}

Sin entrar a valorar las metodologías de obtención de los datos de movilidad usados en el análisis comparativo, las cuales difieren claramente en aspectos técnicos de diseño, muestra y extracción de la información, conviene resaltar que estas fuentes son de muy diferente nivel de detalle y origen. Por un lado, cada una de ellas ha sido capturada en un ámbito temporal diferente. Como es conocido, los hábitos de movilidad de la sociedad actual se caracterizan por una fuerte dependencia respecto a las características socioeconómicas de cada momento y cada lugar. Aun siendo extraídos por la misma metodología, los resultados variarían según el año o incluso el mes de realización del estudio; por lo que el marco de referencia temporal de realización del estudio afecta a los ratios de movilidad de la población. En este sentido, conviene resaltar que la matriz derivada de la encuesta se corresponde con datos tomados en el año 2010 mientras que la matriz derivada de la telefonía 
móvil se asocia al año 2015. Por ello resulta lógico que puedan observarse grandes discrepancias desde el punto de vista numérico, causadas no sólo por cambios de la población (e.g. número de residentes, hábitos de movilidad, etc.), sino por una posible variación de determinadas características de la infraestructura viaria. Por ejemplo, en la zona de transporte de Los Prados se ubican las cocheras de MetroMalaga, que no se encontraban en funcionamiento en el momento de la realización de la encuesta de 2010, pero sí en 2015. Es por ello que la comparativa realizada ha tenido como objetivo disponer de un marco de referencia aproximado para derivar conclusiones.

\subsection{Análisis interno: Debilidades y fortalezas}

Similitud de la información de movilidad contenida en las matrices. Patrones $O-D$

La Tabla 1 presenta los índices de correlación entre la información contenida en las matrices derivadas de ambos tipos de fuentes a nivel de pares O-D, de viajes por orígenes y viajes por destinos, todo ello tanto para la zonificación basada en zona de transporte como en macrozonas. Con el fin de medir el grado de relación numérica entre fuentes, se ha evaluado el coeficiente de correlación lineal (coeficiente de Pearson, $R_{p}$ ). Adicionalmente, dado que las matrices también capturan la interacción espacial entre el origen y el destino del viaje, se ha evaluado un nueva métrica basada en la media del índice de similitud estructural (MSSIM), desarrollado para la comparación de imágenes a nivel de píxel (Wang et al., 2004; Djukic et al., 2013). Su principal ventaja es que incluye en el proceso de evaluación información adicional sobre los patrones estructurales de las matrices. Este índice de correlación estructural varía en el rango $[-1,1]$; siendo el mayor valor positivo un indicador de buena similitud, mientras que el valor negativo más pequeño significa disimilitud (diferencias significativas).

\begin{tabular}{|l|c|c|c|}
\hline \multirow{2}{*}{ Información } & \multirow{2}{*}{ Coeficiente } & \multicolumn{2}{|c|}{ Granularidad } \\
\cline { 3 - 4 } & & Zona de Transporte & Macro-zona \\
\hline Viajes por par $O-D$ & MSSIM $^{(*)}$ & 0.7751 & 0.8326 \\
\cline { 2 - 4 } & $\mathrm{R}_{\mathrm{p}}$ & 0.4361 & 0.8129 \\
\hline Viajes por origen & $\mathrm{R}_{\mathrm{p}}$ & 0.3790 & 0.7968 \\
\hline Viajes por destino & $\mathrm{R}_{\mathrm{p}}$ & 0.3808 & 0.7910 \\
\hline
\end{tabular}

*) Sólo disponible para información en forma de matrices

Tabla 1 - Correlación entre información derivada de telefonía y de encuestas

El análisis de resultados revela una fuerte correlación entre ambas fuentes en cuanto a similitud estructural, tanto a nivel macro-zona como de zona de transporte. Los elevados valores de MSSIM claramente indican que las dos matrices tienen una estructura similar; es decir, que los patrones en la matriz derivada de la telefonía son muy próximos a aquellos capturados por la matriz derivada de encuestas. En cambio, desde el punto de vista numérico, 
el análisis indica que el grado de asociación lineal entre ambas fuentes es débil-medio positivo a nivel de zona de transporte, mientras que a nivel de macrozona se experimenta un incremento sustancial en el grado de correlación. Este comportamiento se mantiene para los totales de viajes por orígenes y por destinos.

Focalizando el análisis a nivel de pares O-D en las diferentes horas del día, se aprecia que el grado de correlación lineal (Figura 1a) durante los períodos de tiempo en los que se concentra la mayor parte del tráfico (7:00 y 20:00 horas), mantiene la tendencia señalada en el análisis diario; débil correlación lineal a nivel de pares de zonas de transporte y fuerte a nivel de pares de macrozonas. Análogamente, en relación con la correlación estructural (Figura 1b) en las diferentes horas del día, se aprecia que ambas fuertes obtienen altos niveles de similitud, tanto a nivel de pares de zonas de transporte como de pares de macrozonas, especialmente en la franja horaria mencionada previamente. Se observa que el índice de similitud estructural (MSSIM) es menos sensible a la zonificación empleada que los coeficientes de correlación lineal, los cuales mejoran significativamente cuando aumenta el nivel de agregación geográfica. Esto se explica porque el índice MSSIM mide aspectos estructurales relativos a la inter-relación entre celdas de la matriz, en lugar del propio valor individual de la celda.

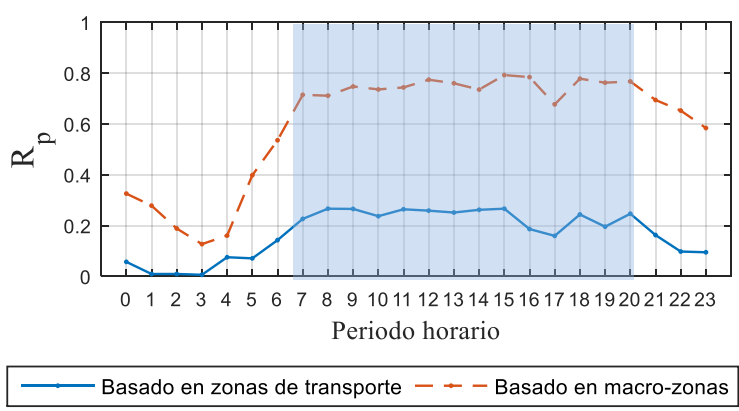

(a)

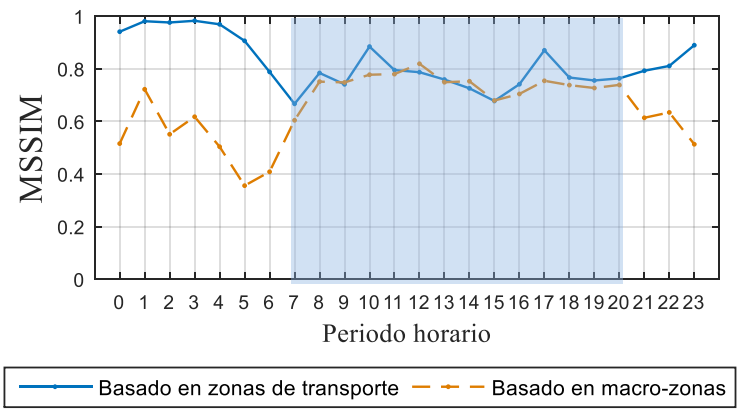

(b)

Fig. 1 - Evolución horaria de la (a) correlación lineal, y (b) estructural de la información de viajes entre orígenes y destinos derivadas de telefonía y encuestas.

Resolución espacial de los eventos móviles

Aunque el modelo de Smart Steps emplea los más novedosos desarrollos en big data, geolocalización y geo-procesos, los eventos generados pueden resultar insuficientes para una estimación precisa de determinados viajes. Estos eventos generan "huellas" que son traducidas a una ubicación "aproximada" que, con la resolución espacial que ofrece esta tecnología, no se corresponde con un punto exacto sino con áreas generales relacionadas con el área de servicio de las antenas de la red de telefonía. En la Figura 2 se muestra un ejemplo con la división de una región de estudio en áreas de cobertura de antenas de telefonía y en zonas basadas en la división administrativa según la zonificación tradicional de transporte. Las áreas de telefonía, definidas mediante diagramas de Voronoi, son variables, siendo pequeñas en zonas densamente pobladas y mucho más grandes en áreas de baja densidad de 
población (Figura 2a). En cambio, la zonificación tradicional usada en estudios de transporte consiste en áreas poligonales correspondientes, por ejemplo, a divisiones administrativas de las ciudades (Figura 2b). Es normal que el área de servicio de una antena no coincida exactamente con una zona de transporte, sino parcialmente con dos o más de ellas (marcado en rojo, Figura 2). El sistema debe seleccionar una sola de ellas, de ahí que aparezca cierto sesgo al establecer la correspondencia entre el área de servicio de las antenas con las zonas de transporte. Además, los sistemas radiantes de emisión empleados en la telefonía móvil contemplan la existencia de solapamiento entre antenas vecinas para evitar "sombras" de cobertura. Por lo que los límites de contorno de la cobertura de una antena no están tan claramente identificados como los de las zonas de transporte. Este error es menor cuanto más extensa es la zona con la que se tiene que emparejar el área de servicio de la antena (marcado en naranja, Figura 2), de ahí que los resultados sean claramente mejores al aumentar el nivel de agregación espacial de la zonificación.

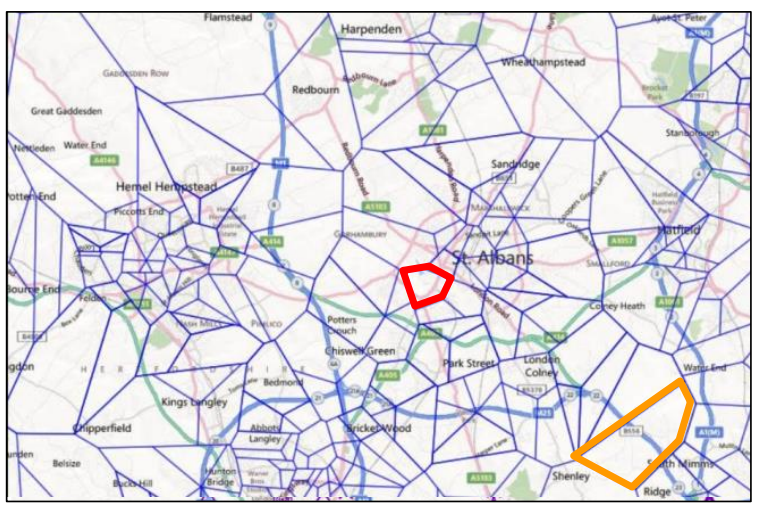

(a)

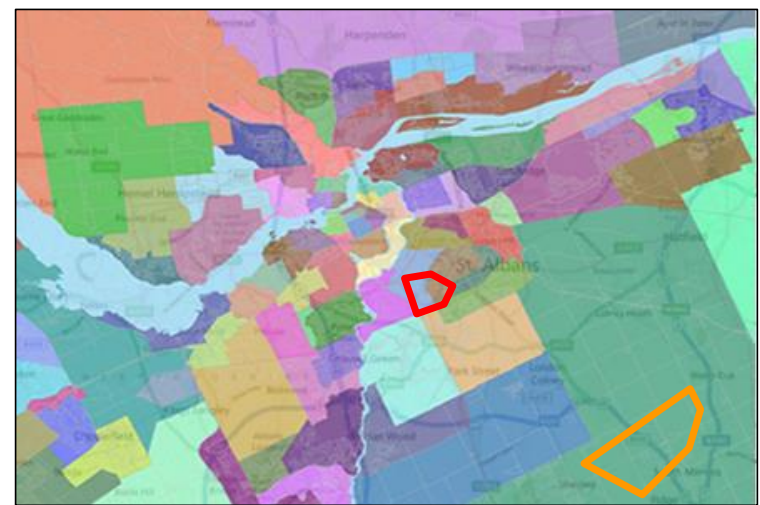

(b)

Fig. 2 - División por (a) áreas de cobertura de antenas de telefonía, y (b) según zonificación tradicional basada en divisiones administrativas; se representan dos ejemplos de correspondencia entre zonas de transporte y áreas de servicio de antenas para diferentes tamaños de agregación espacial.

\section{Detección de viajes de proximidad (corta distancia o corta duración)}

A las divergencias puestas de relieve en la sección precedente, se suma una limitación relacionada con la detección de viajes de proximidad. Lo que define la proximidad no es sólo la distancia que separa un origen de un destino, sino la capacidad que tienen las personas de trasladarse de un punto a otro en un tiempo y en un modo adecuado. Por tanto, el concepto de proximidad incluye tanto el tiempo de viaje como la distancia cubierta en un desplazamiento, conformando variables de la función conocida como "accesibilidad". En base al contraste de fuentes realizado se ha identificado la existencia de unos umbrales técnicos por debajo de los cuales la telefonía móvil carece de las prestaciones necesarias para la detección consistente de desplazamientos. Este aspecto ha puesto de manifiesto al analizar la distribución de viajes según distancia (Figura 3a), identificándose que telefonía presenta dificultades para detectar/caracterizar de un modo preciso los viajes de proximidad, muchos de ellos asociados con distancia de viaje inferior a $2.5 \mathrm{~km}$. Dado que la distancia 
está estrechamente relacionada con el del tiempo de viaje, también existe un tiempo de viaje mínimo de detección, el cual fue establecido por el operador en torno a 15 minutos. En cambio, considerando sólo aquellos desplazamientos de media o larga distancia ( $\geq 2.5 \mathrm{~km})$, los resultados de ambas fuentes son muy similares tanto en la distribución de viajes por distancia (Figura 3a) como en orden de magnitud de las cifras totales (Figura 3b). No obstante, los desplazamientos de proximidad representan un segmento muy significativo de la movilidad cotidiana de una gran ciudad. Las encuestas recogen información de viajes con independencia de este aspecto, de ahí que sigan desempeñando un papel necesario en los estudios de transporte, además de por otras consideraciones que se exponen a continuación.

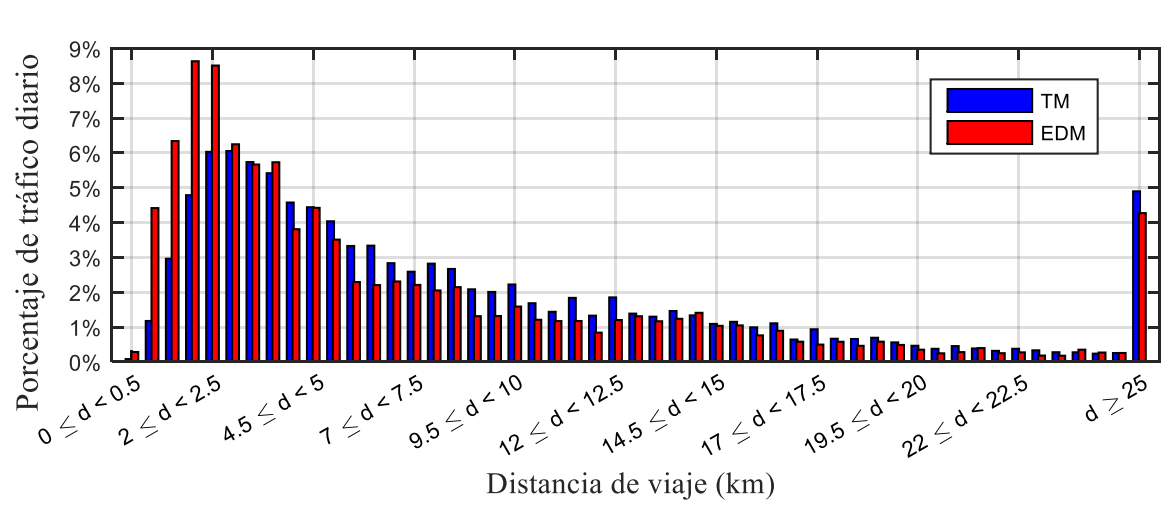

(a)

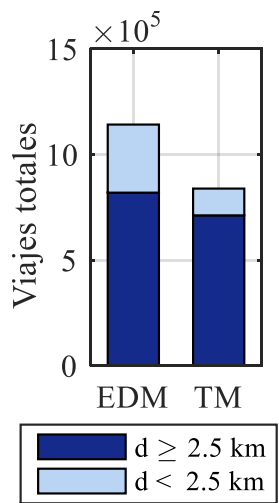

(b)

Fig. 3 - Distribución del número de viajes derivados de la telefonía móvil (TM) y las encuestas (EDM) según (a) la distancia de viaje, $d$, y (b) total de viajes.

\section{Detalle de la información de los viajes mediante encuestas}

Los datos capturados mediante campañas de encuestas a usuarios del sistema de transporte presentan la característica de ofrecer una información exhaustiva sobre la movilidad de éstos, tales como el modo de transporte empleado o el motivo de realización, junto con información socioeconómica de los hogares e individuos encuestados. Estos aspectos resultan muy difíciles de inferir de forma automática con el estado actual de la tecnología móvil. Por otra parte, en el contexto de múltiples paradas de viajes asociados con el encadenamiento de motivos (por ejemplo, dejar a los niños en la escuela antes de ir a trabajar), la tecnología móvil presenta graves dificultades para detectar paradas (o etapas) realizadas durante el viaje, sobre todo si éstas son corta en el tiempo. Por el contrario, una encuesta de viaje es una de las pocas fuentes de datos que recogen dicha información, que pueden ser útiles para construir modelos de planificación específicos. Un análisis exhaustivo de la información capturada por la telefonía móvil podría permitir estimar parcialmente estas características bajo ciertas hipótesis. De hecho, recientemente se han publicado trabajos en la literatura capaces de estimar detalles como el modo de transporte (Wang et al., 2010) o viajes O-D por motivo (Alexander et al., 2015) a partir de datos móviles; sin embargo, las técnicas experimentadas no se encuentran suficientemente desarrolladas para garantizar una explotación de amplio espectro. En este estudio, sin embargo, la matriz derivada de la telefonía móvil usada en el proyecto ha sido un resultado final ya procesado, garantizando la total privacidad de los usuarios de telefonía, al ser el propio operador el encargado de todo 
el proceso de anonimización, agregación, creación de matriz y extrapolación. Pero, como contrapartida, se impide cualquier tratamiento adicional para extraer dichos detalles adicionales, e incluso para validar las metodologías aplicadas en su tratamiento. Adicionalmente la telefonía móvil precisa que el usuario esté "ubicado" más de 30 minutos en una zona para poder discernir las distintas etapas de un viaje. De modo que si un usuario que inició su desplazamiento y se detuvo, comienza nuevamente a moverse pero sin haber transcurrido ese umbral mínimo de tiempo, la metodología del operador considera que el desplazamiento forma parte del mismo viaje. Estos viajes multietapa pueden ocurrir en cualquier momento del día, aunque son más característicos en las franjas de hora punta, donde el usuario combina la movilidad ocupacional (e.g. ir al trabajo o acudir al lugar de estudios) con la movilidad personal (e.g. llevar niños al colegio, acudir al médico o realizar otras gestiones). Estos aspectos, junto con el antes mencionado de viajes de proximidad, motivan la necesidad de continuar realizando encuestas, especialmente para dimensionar este segmento de la movilidad de corta distancia o corta duración. No obstante, las encuestas ya no precisarán la envergadura que han tenido hasta ahora puesto que, comparativamente, los datos de movilidad derivados de la telefonía móvil aportan una serie de mejoras con las que las encuestas difícilmente pueden competir.

Reducción de recursos (tiempo y coste) para captura y procesado de datos con la telefonía Una campaña de encuestación, desde la toma inicial de datos hasta la explotación de los primeros resultados, supone un largo proceso que puede abarcar un periodo de incluso un año. Esta restricción, junto con los costes económicos y los medios necesarios para llevarla a cabo, condiciona su ejecución a periodos de entre 5 y 10 años. Es evidente que durante ese tiempo pueden producirse importantes variaciones de la movilidad debido a cambios socioeconómicos, de infraestructura o sociales. Con lo cual, la percepción de la movilidad reflejada por esos estudios suele quedarse obsoleta al poco tiempo de su elaboración. Es en este ámbito donde aparece una de las principales fortalezas de la telefonía móvil, en el caso concreto de este proyecto, bastaron unas cuantas semanas para disponer de matrices sobre el área de estudio (tanto de laborable como de fin de semana).

Muestra extensa y homogéneamente distribuida por el territorio mediante la telefonía

Así mismo, se ha comprobado que emplear como muestra un conjunto de individuos que llevan teléfono consigo en todo momento permite manejar una muestra extensa y distribuida por el área de estudio. La alta tasa de penetración de la telefonía celular, en España supera ya el 108\% (CNMC, 2015), permite emplear como muestra a un conjunto de usuarios cuyos datos de movilidad son razonablemente representativos de las pautas de movilidad del universo de usuarios. Todo ello sin provocar ningún tipo de perturbación, ya que los datos proceden de eventos generados de forma automática y pasiva por los usuarios de telefonía móvil. Con ello se solventa la dificultad de encontrar personas dispuestas y capaces de ser entrevistadas con respecto a su viaje (uno de los principales obstáculos a los que se enfrentan las campañas de encuestas), así como otros problemas típicos de las encuestas asociados a la no-respuesta y la información no reportada (Ortúzar y Willumsen, 2001). En este estudio, 
se considera que la muestra de la telefonía móvil se corresponde con todo el universo de usuarios en movilidad distribuidos uniformemente sobre el área de estudio conforme a la cuota de mercado del correspondiente operador. En términos de tamaño de la muestra, casi 30.000 personas fueron entrevistadas para construir las matrices (en torno al 3\% de la población); mientras que las matrices derivadas de la telefonía se calcularon sobre una base de aproximadamente 200.000 personas, un valor claramente superior. De hecho, emplear como muestra a usuarios móviles permite incluir no sólo a residentes en el área de estudio, sino a visitantes temporales que también influyen en la demanda de transporte en la región considerada. No obstante, atendiendo a las características de la muestra, conviene resaltar que la movilidad recogida por la telefonía no monitoriza a menores de edad (por razones de protección de datos), mientras que las encuestas sí que lo hacen (parcialmente por encima de una cierta edad).

Mayor cobertura de relaciones $O-D$

La gran representatividad de la muestra basada en la telefonía, relacionada con la cuota de penetración del operador, se traduce en una captura de más relaciones o pares O-D que conforman la matriz. Este aspecto es mostrado en la Figura 4, representado en negro aquellos pares O-D en los que se ha observado al menos un viaje en los pares de las matrices derivadas de cada una de las fuentes; los espacios en blanco identifican los pares en los que no se detecta movilidad. Con un sistema de zonificación de 178 zonas de transporte, la matriz se compone de 31506 pares de viajes inter-zonales.

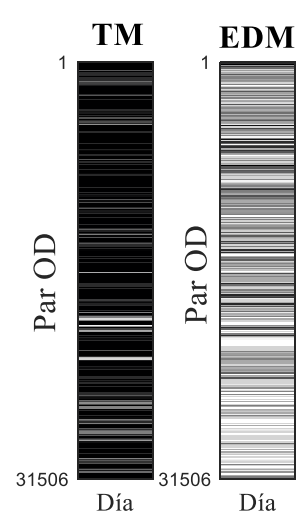

(a)
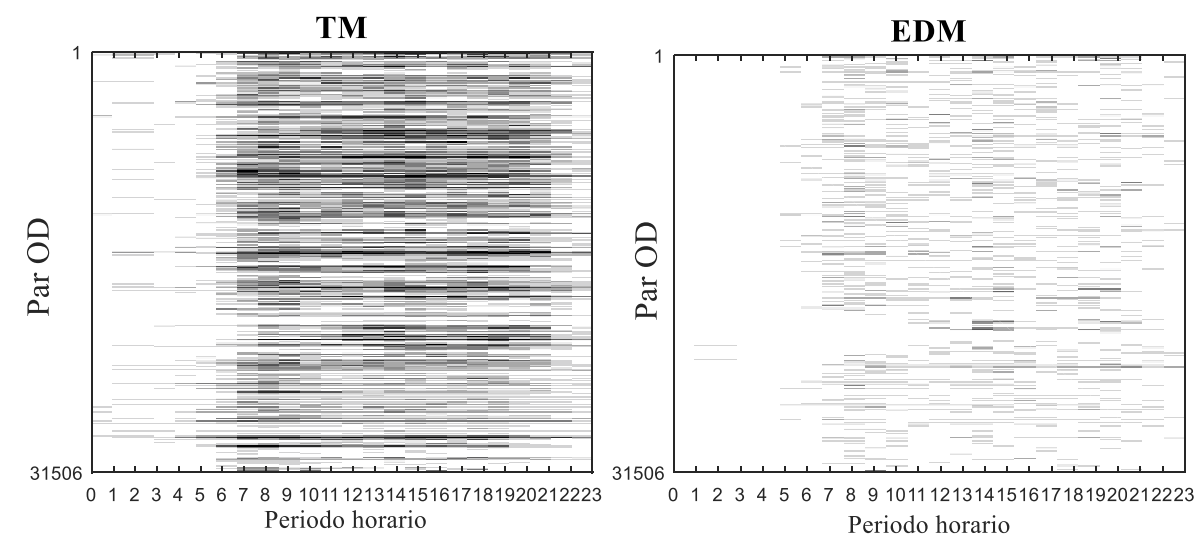

(b)

Fig. 4 - Pares O-D observados por la telefonía (TM) y encuestas a nivel diario y horario (EDM).

La comparativa a nivel diario (Figura 4a) pone de manifiesto que la telefonía móvil captura la movilidad en un porcentaje muy alto de pares O-D (89\%), los cuales son visiblemente superiores a los recogidos por la matriz diaria asociada a encuestas (34\%). Esta diferencia se mantiene en nivel horario (Figura 4b); por ejemplo, en el período horario de las 8 horas (viajes en la franja 08:00-8:59), el porcentaje de pares observados en la matriz derivada de la telefonía se sitúa en torno al 32\%; frente a menos del 10\% para la matriz horaria derivada 
de la encuesta.

\subsection{Análisis externo: amenazas y oportunidades}

La telefonía móvil es un segmento de la tecnología que ha evolucionado con gran rapidez en los últimos años. La complejidad de los hábitos de vida modernos plantea enormes retos y oportunidades para esta tecnología. La posibilidad de recopilar más datos y de mayor calidad y frecuencia ha motivado el lanzamiento de numerosos estudios piloto. Por ello, resulta difícil identificar alguna amenaza que impida el uso de esta tecnología en futuros estudios de movilidad. Sólo algún cambio legislativo que introduzca alguna restricción adicional puede constituir amenaza a su avance. En todo caso, la privacidad es un aspecto atendido con sumo cuidado y no debe suponer un límite para el auge de la telefonía. Cabe destacar que todo el procesado y explotación de los eventos móviles han sido tratados de forma agregada y anónima con el fin de respetar la normativa vigente de protección de datos. A medida que estas tecnologías avancen y los estudios pilotos ganen experiencia, su adopción se extenderá rápidamente en el ámbito del transporte, replicando los modelos puestos en marcha con éxito en otras ciudades del mundo.

En la actualidad, aunque la realización de encuestas de movilidad está sujeta a fuertes controles de calidad para regular su fiabilidad, en todas las ocasiones se introduce cierto sesgo debido al diseño, selección o determinación del tamaño de la muestra que la genera. Siempre se presenta el caso de individuos que, participando en la movilidad de una región, no son entrevistados, bien por rechazar su participación o bien por tratarse de visitantes que realizan viajes esporádicos. La utilización de información derivada de la telefonía móvil permite capturar esos segmentos de la movilidad, mediante una muestra sustancialmente más representativa a nivel cuantitativo (mayor tamaño muestral relacionado con la cuota del operador) y cualitativo (con usuarios que realmente participan en la movilidad del área en un periodo de tiempo concreto), solventando problemas de baja tasa de respuesta o sesgo.

Otro aspecto desprendido del análisis se centra en la captura de la movilidad en zonas de poca o nula población, como el caso de polígonos industriales, estaciones centrales de autobús y tren o incluso el aeropuerto. Se comprobó que para aquellas zonas sin población (Figura 4), la telefonía captura una cantidad sustancialmente mayor de viajes que las encuestas, probablemente debido a errores de diseño de la muestra entrevistada y/o representatividad de la zona. Las encuestas domiciliarias recopilan viajes de residentes en el área de estudio; las zonas no pobladas están excluidas del marco de muestreo (no hay hogares para ser encuestados). Ello sugiere la posibilidad de que la muestra tomada en las encuestas pueda no ser representativa de todas las personas que viajan desde o hacia estas zonas sin población. Existe otro tipo de encuestas (e.g. encuestas de interceptación) que se realizan a "pie de calle" con objeto de interceptar desplazamientos de llegada o salida del área de estudio, o incluso para medir los viajes de los no-residentes. Se emplean para verificar y ampliar la información relativa a viajes procedentes de encuestas domiciliarias, pero exigen la dedicación de recursos adicionales que se suman a los ya empleados en la encuesta 
domiciliaria. Además, aunque este tipo de encuestas puede sintetizar mejor la movilidad sobre estas zonas sin población, se utiliza un muestreo por cuotas no controladas que también presenta problemas de sesgo. Es necesario el uso de una combinación de diferentes métodos de muestreo para aprovechar las ventajas de uno y otro método; pero incluso una combinación de encuestas domiciliarias y encuestas de interceptación no sería capaz de producir matrices donde todas las relaciones O-D hayan sido incluidas en la muestra (Ortúzar y Willumsen, 2001). Sin embargo, la representatividad de la muestra basada en usuarios de la telefonía móvil captura viajes con independencia de las características socioeconómicas de la zona de transporte origen.

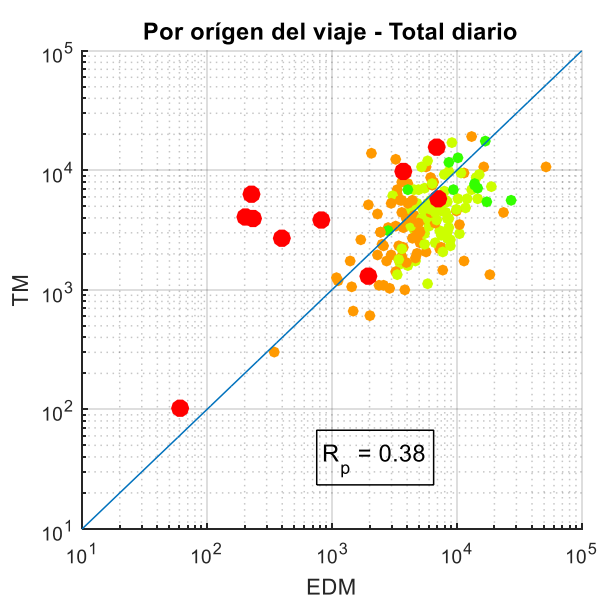

(a)

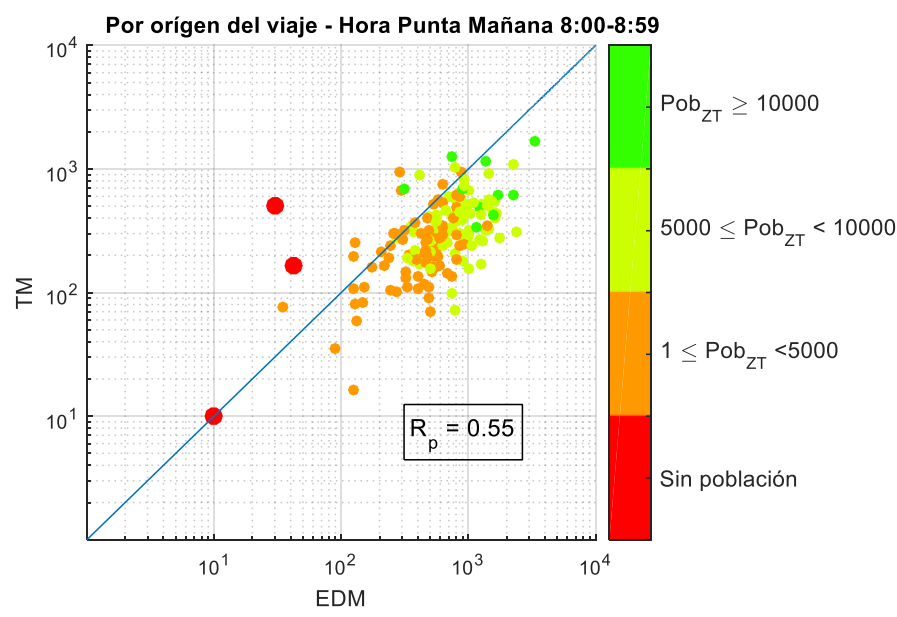

(b)

Fig. 4. Comparativa de viajes originados en cada zona de transporte (telefonía vs. encuestas), por colores según población: (a) total diario y (b) periodo 8:00-8:59.

Adicionalmente, el hecho de disponer de información en un corto plazo permite actualizar de un modo más continuo la información de movilidad disponible de una zona, permitiendo no sólo identificar la realidad actual de la dinámica de un área sino abordar temas a los que hasta ahora se ha dedicado pocos recursos, como la movilidad de fin de semana. Manejar información tan actualizada permite optimizar la gestión de la infraestructura de un modo más dinámico y realista. Cualquier cambio de política de transporte/infraestructura puede ser evaluado en un plazo de tiempo considerablemente reducido, sin tener que esperar a la realización de un costoso nuevo estudio, permitiendo con ello, a su vez, dar respuesta a otros problemas derivados del incremento de tráfico experimentado en las áreas metropolitanas como la saturación y la seguridad.

\section{CONCLUSIONES MÁS RELEVANTES}

En base a todos los aspectos anteriormente comentados, junto a los análisis y contrastes llevados a cabo en el ámbito de este estudio, se pone de manifiesto el alto potencial que ofrece la telefonía en los estudios de transporte. Aspectos como la gran representatividad de la información, en términos de tamaño de muestra de la que se captura la información (aunque limitada a mayores de edad por razones de protección de datos), y el reducido 
tiempo de procesado, lo cual conlleva el uso de datos actuales de movilidad, posicionan a la telefonía móvil como una tecnología prometedora a usar en los estudios de movilidad. Sin embargo, se ha identificado la existencia de unos umbrales técnicos en la telefonía que limitan la detección/caracterización precisa de viajes de proximidad (corta distancia o corta duración). Con el estado actual de la tecnología, existe cierta dificultad para detectar desplazamientos de distancia inferior a $2.5 \mathrm{~km}$, muchos de ellos asociados a duración inferior a 15 minutos. Debido a que este tipo de desplazamientos representa un porcentaje muy elevado de la movilidad cotidiana de una ciudad, las encuestas siguen desempeñando un papel relevante aunque no de la envergadura y coste (económico y temporal) que han tenido en los tradicionales estudios. Conviene enfatizar el hecho que las encuestas facilitan el enriquecimiento de la información de movilidad con otras características adicionales del desplazamiento, tales como motivo, frecuencia, o incluso modo, los cuales no pueden deducirse de forma directa y automática con el estado actual de la tecnología móvil.

A pesar de ello, es indudable que las matrices derivadas de la telefonía móvil constituyen un razonable punto de partida para la generación rápida, extensa y eficiente de matrices. Pero será la aplicación cooperativa de todas las fuentes de información disponibles la que permita obtener resultados de calidad. Con técnicas de optimización y herramientas de modelado se podrán minimizar las imprecisiones derivadas de la telefonía, especialmente en lo referente a viajes de proximidad. En el futuro próximo, cuando la tecnología móvil también incorpore eventos de datos $2 \mathrm{G}, 3 \mathrm{G}$ y $4 \mathrm{G}$ (e.g. email, whatsapp, etc.), la capacidad de este tipo de herramientas mejorará sustancialmente; dando lugar a que los eventos que permiten la identificación de viajes vean incrementada la resolución temporal (los terminales interactúan con dichas redes con mayor frecuencia) y espacial (se trabaja con celdas de menor tamaño).

\section{AGRADECIMIENTOS}

Este trabajo se ha desarrollando gracias a la financiación del proyecto FEDER de la Unión Europea con título "Dinámica de una ciudad. Caracterización de la Movilidad Urbana mediante Datos de telefonía móvil y otras fuentes de información heterogénea" (DIURMOVIL) del Programa Operativo FEDER de Andalucía 2011-2015. Asimismo, se desea mostrar agradecimiento a la Agencia de Obra Pública (Junta de Andalucía, Consejería de Fomento y Vivienda), al Consorcio de Transporte Metropolitano del Área de Málaga, y a Telefónica. Por último, uno de los autores (N. Cáceres) también agradece al Ministerio de Economía y Competitividad el soporte proporcionado a través del Programa de Ayudas Torres-Quevedo (PTQ-13-06428).

\section{REFERENCIAS}

ALEXANDER, L.P., JIANG, S., MURGA, M., GONZALEZ, M.C. (2015). Origindestination trips by purpose and time of day inferred from mobile phone data, Transportation Research Part C, 58(2), pp. 240-250.

AOPJA (2015). DIURMOVIL: Dinámica de una ciudad. Caracterización de la Movilidad Urbana mediante Datos de telefonía móvil y otras fuentes de información heterogénea. 
Agencia de Obra Pública. Junta de Andalucía. Proyecto G-GI3001IDIK. Programa Operativo FEDER, Unión Europea, 2011-2015.

CNMC (2015). Comisión Nacional de los Mercados y la Competencia. Datos Estadísticos sobre Comunicaciones Móviles. http://data.cnmc.es/datagraph/jsp/inf_trim.jsp.

DJUKIC, T; HOOGENDOORN, SP; LINT, JWC VAN. (2013) Reliability assessment of dynamic O-D estimation methods based on structural similarity index. Transportation Research Board, 92nd Annual Meeting, paper no. 13-4851.

GRANDES ÁREAS URBANAS ESPAÑOLAS (2012). D.G de Arquitectura, Vivienda y Suelo. S.G.T., Centro de publicaciones, M. Fomento, España. Año de Publicación: 2013.

ORTUZAR J. DE D., WILLUMSEN L.G. (2001) Modelling Transport. Third Edition. John Wiley and Sons, Chichester.

SMART STEPS - Telefonica Dynamic Insights, http://dynamicinsights.telefonica.com/ WANG, Z., BOVIK, A. C., SHEIKH, H. R., SIMONCELLI, E. P. (2004). Image quality assessment: From error measurement to structural similarity. IEEE Transactions on Image Processing, 13(1), pp.600-612.

WANG, H., CALABRESE, F., DI LORENZO, G., RATTI, C. (2010). Transportation mode inference from anonymized and aggregated mobile phone call detail records. Procedings of 13th International IEEE Conference on Intelligent Transportation Systems, pp. 318-323. 